\title{
Microneedling and Its Use in Hair Loss Disorders: A Systematic Review
}

Robert S. English Jr. (D) · Sophia Ruiz · Pedro DoAmaral

Received: November 8, 2021 / Published online: December 1, 2021

(c) The Author(s) 2021

\section{ABSTRACT}

Introduction: Microneedling (MN) is a minimally invasive procedure involving the induction of percutaneous wounds with medicalgrade needles. In this literature review, we investigate clinical data on $\mathrm{MN}$ for the treatment of hair loss disorders.

Methods: A literature search was conducted through PubMed up to November 2021 to identify original articles evaluating the use of $\mathrm{MN}$ on hair loss disorders. The database was searched using the following keywords: "microneedling," "micro needling," "micro needle," "microneedle," "needle," "dermaroller" and "alopecia," "hair loss," "alopecia," "areata," "cicatricial," or "effluvium."

Results: A total of 22 clinical studies featuring 1127 subjects met our criteria for inclusion. Jadad scores ranged from 1 to 3 , with a mean of 2. As an adjunct therapy, $\mathrm{MN}$ improved hair parameters across genders and a range of hair loss types, severities, needling devices, needling depths of $0.50-2.50 \mathrm{~mm}$, and session frequencies from once weekly to monthly. Across 17

Supplementary Information The online version contains supplementary material available at https:// doi.org/10.1007/s13555-021-00653-2.

R. S. English Jr. ( $₫)$ · S. Ruiz · P. DoAmaral

Perfect Hair Health, 2021 Fillmore, Ste 98, San

Francisco, CA 94115, USA

e-mail: rob@perfecthairhealth.com investigations totaling 911 androgenic alopecia (AGA) subjects, $\mathrm{MN}$ improved hair parameters when paired with 5\% minoxidil, growth factor solutions, and/or platelet-rich plasma (PRP) topicals, or when introduced to subjects whose hair count changes had plateaued for $\geq 6$ months on other treatments. Across four investigations on 201 alopecia areata (AA) subjects, MN improved hair parameters as a standalone therapy versus cryotherapy, as an adjunct to 5-aminolevulinic acid and photodynamic therapy, and equivalently when paired with topical PRP versus carbon dioxide laser therapy with topical PRP. Across 657 subjects receiving $\mathrm{MN}$, no serious adverse events were reported.

Conclusions: Clinical studies demonstrate generally favorable results for $\mathrm{MN}$ as an adjunct therapy for AGA and AA. However, data are of relatively low quality. Significant heterogeneity exists across interventions, comparators, and $\mathrm{MN}$ procedures. Large-scale randomized controlled trials are recommended to discern the effects of $\mathrm{MN}$ as a standalone and adjunct therapy, determine best practices, and establish long-term safety.

Keywords: Microneedling; Alopecia; Hair loss 


\section{Key Summary Points}

Why carry out this study?

There is growing interest in the use of microneedling as a standalone and adjunct therapy for hair loss disorders.

This literature reviews summarizes a body of clinical evidence on microneedling for hair loss disorders to evaluate hair loss outcomes, evidence quality, limitations in research, and areas of opportunity for future investigations.

\section{What was learned from the study?}

Microneedling improves hair loss parameters across a range of hair loss types, needling devices, needling depths, session frequencies, and combination therapies.

While evidence suggests that microneedling might improve hair loss, clinical data are of relatively low quality. With better study designs and efforts to standardize best practices, microneedling could become a staple adjuvant to US Food and Drug Administration (FDA)approved hair loss treatments.

\section{INTRODUCTION}

Alopecia is a common cosmetic concern affecting over $50 \%$ of adults throughout a lifetime [1]. Hair loss disorders are typically categorized into scarring and nonscarring alopecias, with treatments dependent on the pathogenesis and diagnosis determined during dermatological evaluation [2]. While drug and nondrug interventions often help to improve many hair loss disorders, treatments for androgenic alopecia (AGA) are typically relegated to stopping the progression of the condition [3]. Moreover, treatments for alopecia areata (AA) and alopecia totalis (AT) remain limited, with recurrence rates high [4]. Consequently, there remains demand for novel and effective hair loss treatments.

Microneedling $(\mathrm{MN})$ is a minimally invasive procedure involving the induction of percutaneous wounds with $0.25-5.00 \mathrm{~mm}$ medicalgrade needles. First described by Orentreich in 1995 for the use of wrinkles and atrophic scars, MN purportedly releases platelet-derived growth factor and vascular endothelial growth factor to promote wound-healing responses, improve angiogenesis, and attenuate or partially reverse fibrosis resulting from acute injury and skin aging $[5,6]$. MN can be administered at-home or in-clinic, with devices ranging from needling stamps, manual rollers, and automated pens with or without fractional radiofrequency. Across a range of devices, needling depths, and session frequencies, $\mathrm{MN}$ has demonstrated clinical improvements as a standalone and/or adjunct therapy for patients with atrophic scars, actinic keratoses, and pigmentation disorders such as vitiligo and melasma $[6,7]$.

In the last decade, studies have demonstrated that $\mathrm{MN}$ may enhance transdermal delivery, promote anagen-initiating $\mathrm{Wnt} / \beta$ catenin signaling, and improve dermal papillae stem cell proliferation-thus potentiating therapeutic effects for a variety of hair loss disorders [8-10]. In 2013, the landmark study by Dhurat et al. on 100 AGA subjects found that over a 12-week period, once-weekly $\mathrm{MN}$ combined with twice-daily 5\% minoxidil increased hair counts significantly versus minoxidil monotherapy [11]. Since then, investigators have continued to assess the effects of MN as both a standalone and adjunct therapy for hair loss.

In this systematic review, we investigate the use of $\mathrm{MN}$ as a standalone, adjunct, and comparator therapy on hair loss disorders. We evaluate patient populations, interventions, comparators, MN procedures, outcomes, and adverse events-as well as evidence quality using Jadad scoring. We discuss possible mechanisms by which MN may improve hair loss disorders as a monotherapy and an adjunct intervention. Finally, we identify limitations in 
the current body of research and provide recommendations for future clinical trials.

\section{METHODS}

\section{Literature Search}

A broad literature search was conducted through PubMed up to November 2021 to identify original articles that evaluate the use of MN on hair loss disorders. The database was searched using combinations of the following keywords: "microneedling," "micro needling," "micro needle," "microneedle," "needle," "dermaroller" and "alopecia," "hair loss," "alopecia," "areata," "cicatricial," or "effluvium." This article is based on previously conducted studies and does not contain any new studies with human participants or animals performed by any of the authors.

\section{Inclusion and Exclusion Criteria}

All search hits were screened and examined for relevant titles and abstracts. Full texts were reviewed to determine eligibility. Articles were included if they featured all of the following: (a) human subjects with scalp hair loss, (b) MN as a standalone or adjunct therapy, and (c) endpoint measurements related to scalp hair. Articles were excluded if they did not feature (a) original data, (b) human data, (c) endpoint measurements for hair parameters, and/or (d) designs that adequately evaluated the effects of $\mathrm{MN}$ on hair. This literature review is based on previously conducted studies and does not contain any new studies with human participants or animals performed by any of the authors. Full inclusion and exclusion criteria can be found in Table 1 .

RE and SR each independently identified 367 records for screening. RE, SR, and $\mathrm{PD}$ each independently screened all 367 titles and abstracts to assess eligibility, and the 42 full texts to determine inclusion. RE, SR, and PD each independently assessed Jadad scores. Any disagreements in identifications, screenings,
Table 1 PICOS inclusion and exclusion criteria

\begin{tabular}{|c|c|c|}
\hline Parameter & Inclusion criteria & Exclusion criteria \\
\hline Patients & $\begin{array}{l}\text { Patients of any age } \\
\text { treated for scalp } \\
\text { hair loss }\end{array}$ & \\
\hline Intervention & $\begin{array}{l}\text { MN as a standalone } \\
\text { or adjunct therapy }\end{array}$ & $\begin{array}{l}\text { MN devices with } \\
\text { needle-releasing } \\
\text { drugs, acupuncture } \\
\text { needles }\end{array}$ \\
\hline Comparator & $\begin{array}{l}\text { How effective is } \\
\text { MN at improving } \\
\text { hair loss } \\
\text { outcomes? }\end{array}$ & \\
\hline Outcomes & $\begin{array}{l}\text { Primary endpoints: } \\
\text { phototrichogram, } \\
\text { investigator, and/ } \\
\text { or patient } \\
\text { assessments }\end{array}$ & $\begin{array}{l}\text { Any study not } \\
\text { designed to } \\
\text { adequately test for } \\
\text { the standalone or } \\
\text { additive effect of } \\
\text { MN }\end{array}$ \\
\hline Study design & Prospective studies & $\begin{array}{l}\text { Retrospective design, } \\
\text { case series, } \\
\text { literature reviews, } \\
\text { or nonhuman } \\
\text { subjects; studies } \\
\text { with fewer than } \\
\text { five patients; } \\
\text { ongoing clinical } \\
\text { trials; Jadad scores } \\
\text { lower than } 1\end{array}$ \\
\hline
\end{tabular}

A table summarizing the inclusion and exclusion criteria in our systematic review for clinical studies investigating the use of $\mathrm{MN}$ for the treatment of hair loss disorders

selections, and/or Jadad scores were discussed by $\mathrm{RE}$ and PD and resolved by RE.

\section{RESULTS}

Of the 42 full texts accessed to assess eligibility, 20 were excluded on the basis of the wrong intervention $(n=2)$, outcome $(n=4)$, or study 


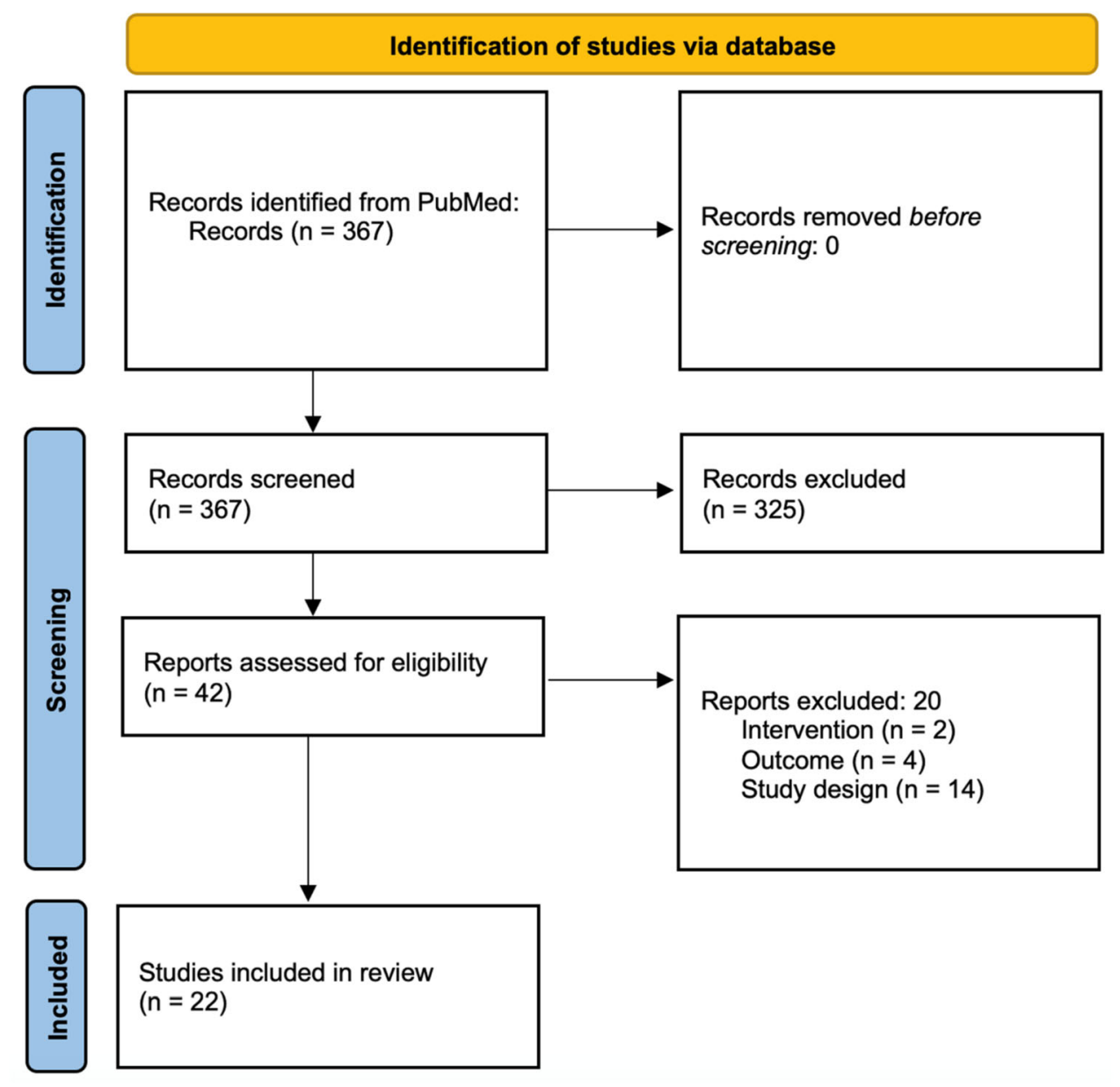

Fig. 1 PRISMA flowchart. A PRISMA flowchart detailing the process of eligibility for all records reviewed for the literature review, as well as the number of studies identified, screened, excluded, and included

design $(n=14)$. A total of 22 clinical studies met our criteria for inclusion: 17 trials with randomization and 5 nonrandomized prospective cohorts (Fig. 1). Jadad scores ranged from 1 to 3 , with a mean score of 2 (Table S1).

Of the 22 studies, 16 were conducted on AGA subjects, 4 on alopecia areata subjects, 1 on alopecia totalis subjects, and 1 on both AGA and telogen effluvium (TE) subjects. A total of 1127 subjects ( 856 males and 269 females) were included featuring the following hair loss types: AGA $(n=911)$, AA $(n=201)$, AT $(n=8)$, and TE $(n=7)$ [11-32].

\section{AGA}

\section{Patients}

Within studies featuring AGA subjects, enrollment ages ranged from 18 to 70 years, with a subject-weighted average of 33.75 years. Of the 15 studies with male AGA subjects, 1 did not enroll subjects based on a classification system, while 14 included males with hair loss based on the Norwood-Hamilton scale: I $(0.0 \%)$, II (46.7\%), III (93.3\%), IV (93.3\%), V (60\%), and VI (26.7\%). Of the seven studies with female AGA subjects, one did not enroll subjects based on any classification system, one enrolled based on Sinclair scores, and five enrolled females with hair loss determined by the Ludwig scale: I (80.0\%), II (60.0\%), and III (60.0\%) (Table 2). 


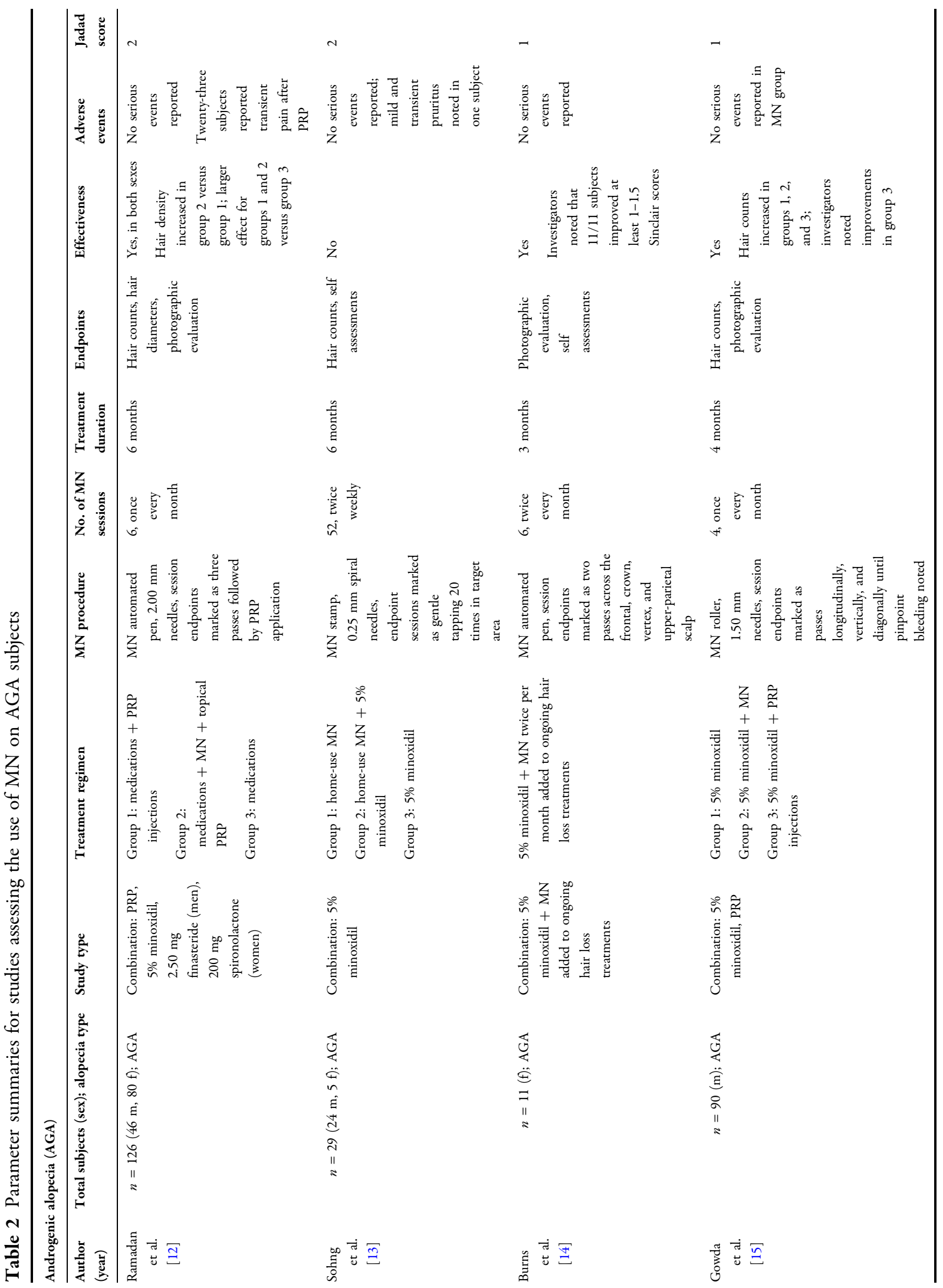




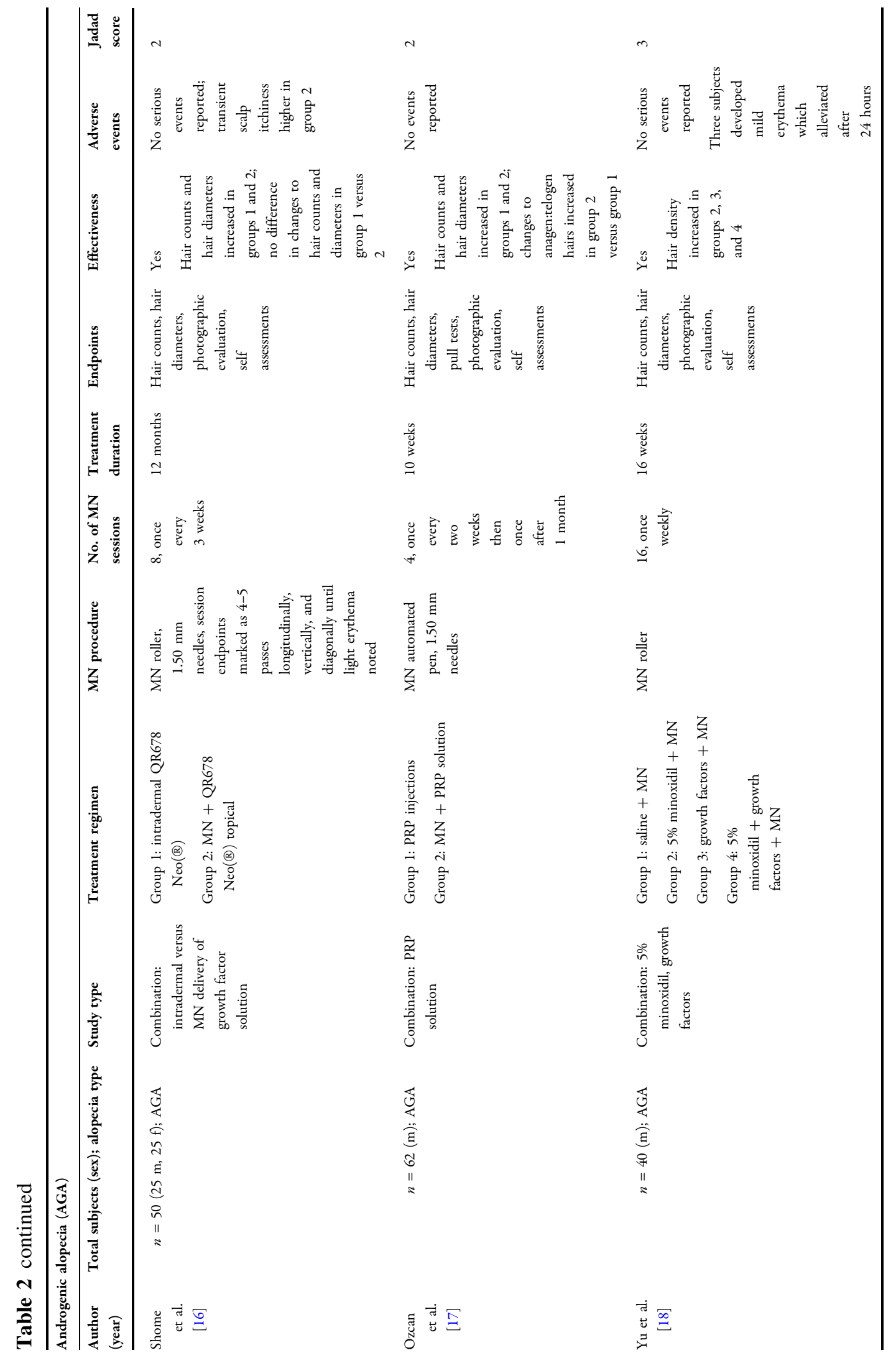




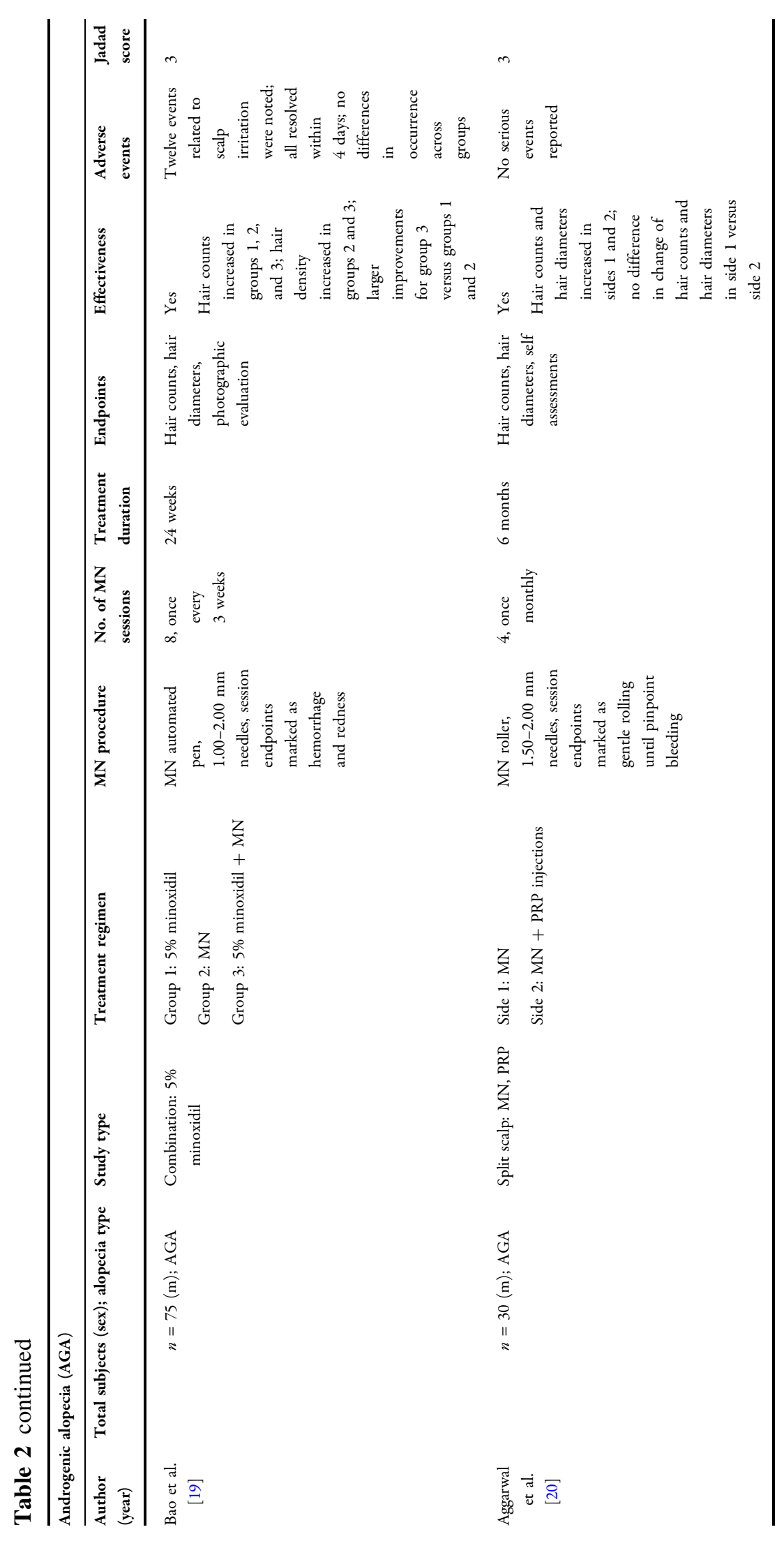




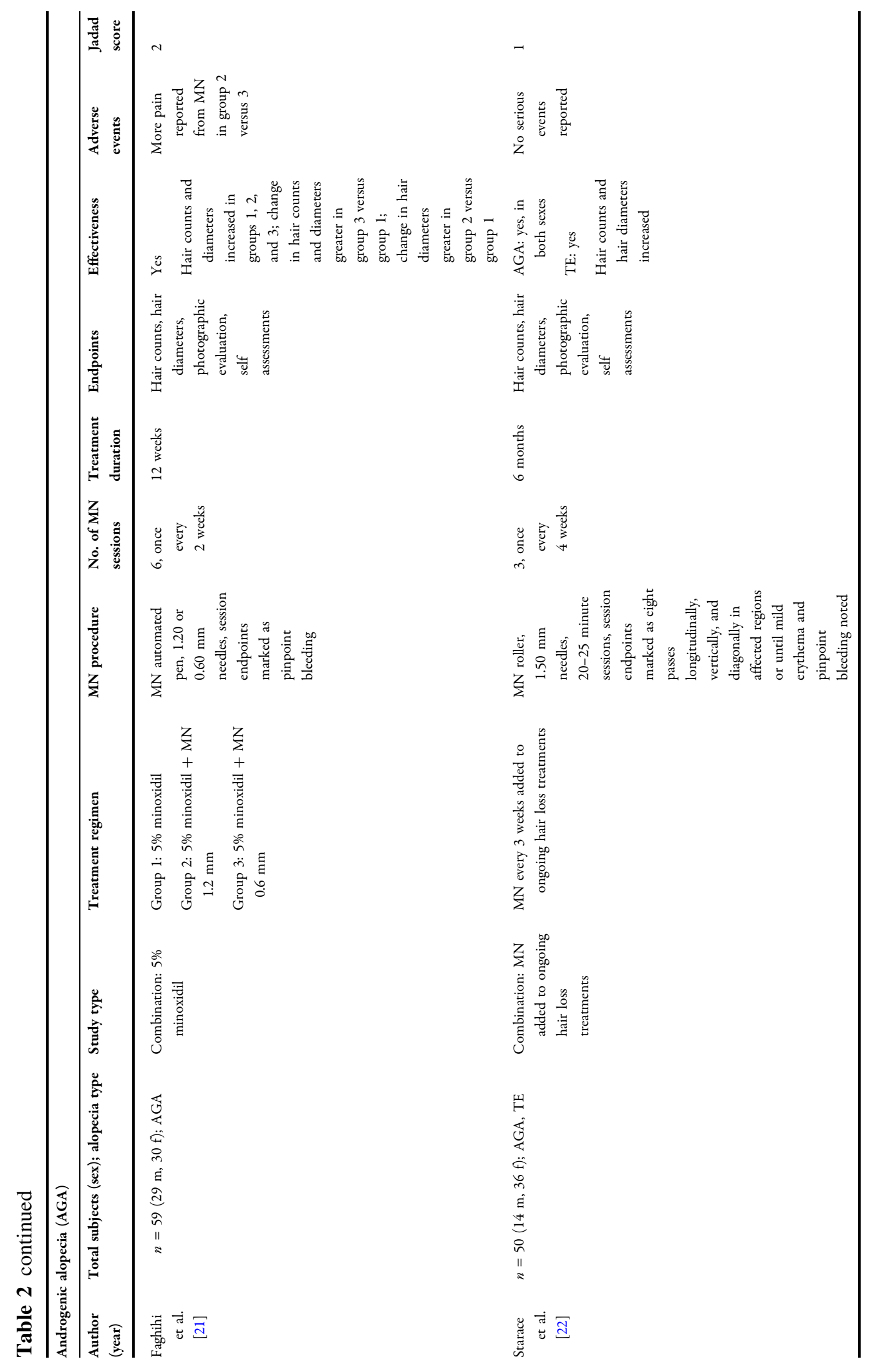




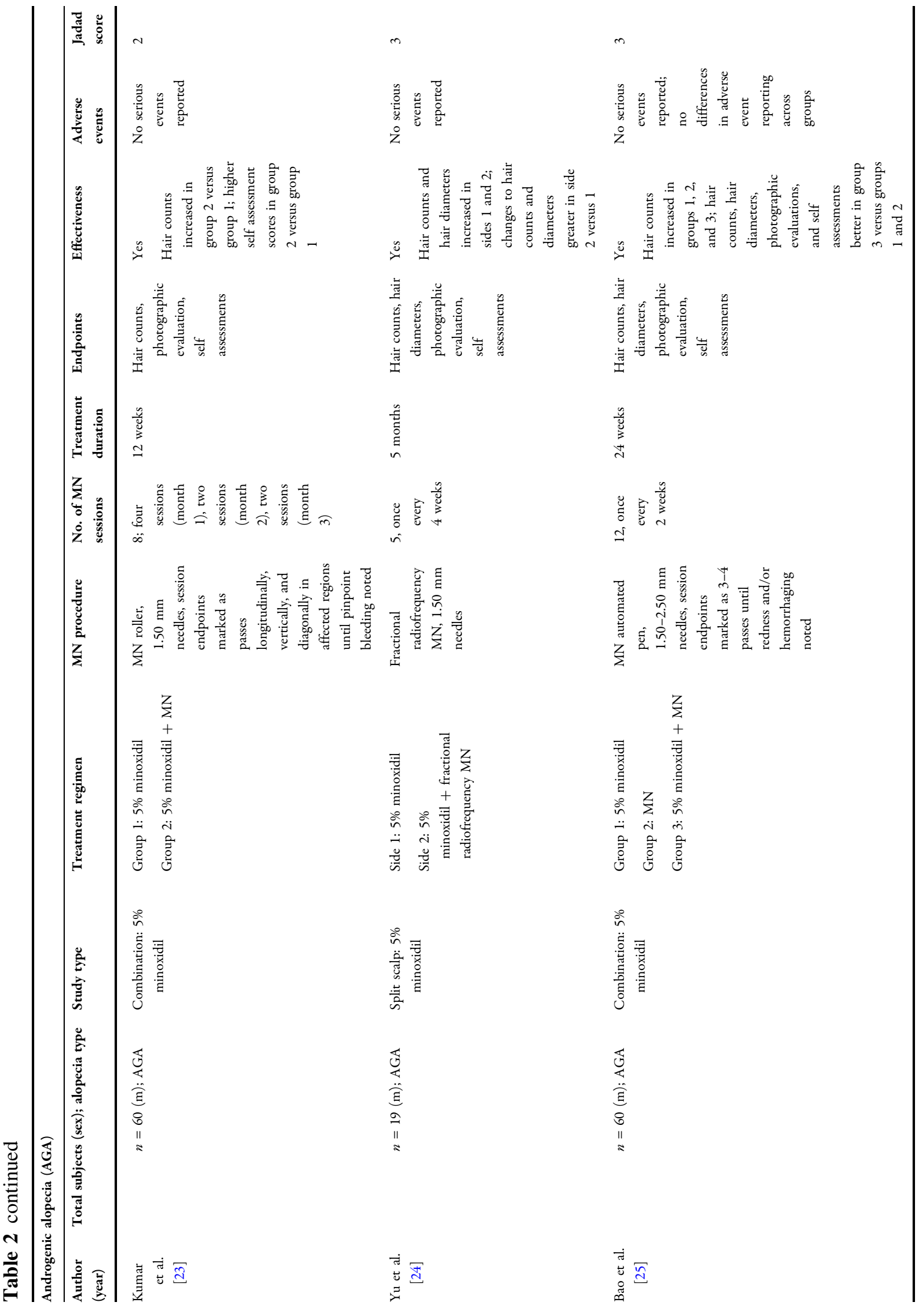




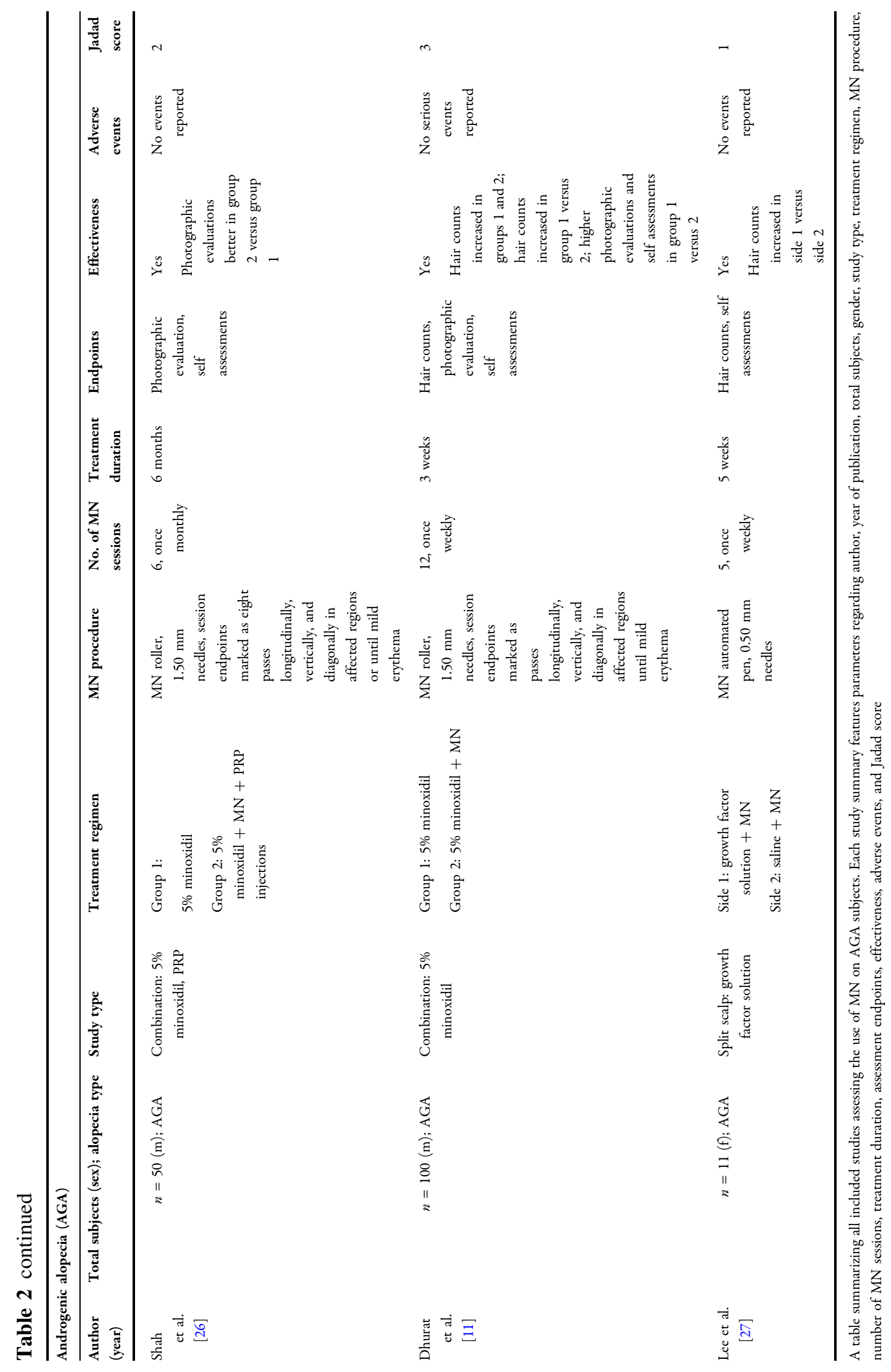




\section{Interventions and Comparators}

In total, 536 subjects received MN therapy, while 375 received other hair loss interventions. Across all study groups, MN was included as a standalone therapy in 6 groups $(n=105)$. As an adjunct therapy, $\mathrm{MN}$ was evaluated alongside topical minoxidil in 10 groups $(n=234)$, proprietary topicals and/or growth factor solutions in 3 groups $(n=46)$, PRP in 2 groups $(n=61)$, continued medication use in 1 group $(n=50)$, PRP and systemic medications in 1 group $(n=42)$, PRP with topical minoxidil in 1 group $(n=25)$, topical minoxidil and continued medications in 1 group $(n=11)$, and topical minoxidil alongside growth factors in 1 group $(n=10)$ (Table 2).

\section{MN Procedures}

MN devices tested included manual rollers $(n=8)$, automated pens $(n=7)$, manual stamps $(n=1)$, and automated fractional radiofrequency pens $(n=1)$. Needle lengths ranged from 0.25 to $2.50 \mathrm{~mm}$, with a mean needle length of $1.39 \mathrm{~mm}$.

The frequency of $\mathrm{MN}$ sessions ranged from twice weekly to once monthly, with a mean session frequency of once per 2.64 weeks. The number of $\mathrm{MN}$ sessions ranged from 3 to 52 , with a mean of $9.53 \mathrm{MN}$ sessions per study. Treatment durations averaged 20.01 weeks.

While four studies did not specify $\mathrm{MN}$ session endpoints, 13 studies standardized endpoints by a number of passes, directions, and/or taps $(n=3)$, mild erythema $(n=3)$, a number of passes and/or bleeding $(n=3)$, or passes until hemorrhage $(n=4)$ (Table 2$)$.

\section{Outcomes}

In total, 15 of 17 studies assessed hair parameters through phototrichograms (i.e., hair counts, hair diameters, and/or hair densities). Of the 17 studies, 6 included MN-only groups, whereas all studies tested $\mathrm{MN}$ alongside other hair loss interventions.

Of the six MN monotherapy groups, two noted significant increases to total hair counts, one found significant increases to hair diameters and total hair density, and three showed no effect $[13,18-20,25,27]$.
Of the seven studies testing MN with 5\% minoxidil, six found statistically significantly increased hair counts versus 5\% minoxidil alone, and for a range of devices and needle lengths: rollers, automated pens, and fractional radiofrequency devices with depths from 0.60 to $2.50 \mathrm{~mm}[11,15,18,19,21,23,25]$. However, Sohng et al. tested 5\% minoxidil with a $0.25 \mathrm{~mm}$ needling stamp twice-weekly and found no effect on hair parameters [13]. When comparing $5 \%$ minoxidil with $0.60 \mathrm{~mm}$ or $1.20 \mathrm{~mm}$ needle lengths, Faghihi et al. found that $0.60 \mathrm{~mm}$ needle lengths led to significant hair count and diameter increases versus 5\% minoxidil, whereas $1.20 \mathrm{~mm}$ needle lengths only saw hair count increases versus 5\% minoxidil [21].

All three studies testing MN alongside proprietary topicals and/or growth factors noted increases to hair counts $[16,18,27]$. Lee et al. and $\mathrm{Yu}$ et al. demonstrated improved hair parameters but no differences across groups when comparing $\mathrm{MN}$ use with topical versus intradermal delivery of proprietary products and/or growth factors [18, 27]. Conversely, Ozcan et al. found that $\mathrm{MN}$ alongside either topical or injectable PRP significantly increased hair counts and diameters similarly across groups, but that subjects receiving $\mathrm{MN}$ alongside topical PRP saw greater improvements to anagen:telogen hairs [17]. Interestingly, in a split-scalp study, Aggarwal et al. showed that both MN and MN with PRP injections increased hair diameters and density equivalently-with no significant differences noted across groups [20].

Two studies tested the introduction of MN alongside ongoing hair loss medications $[14,22]$. Burns et al. found that twice monthly MN combined with 5\% minoxidil improved Ludwig scores for 11/11 females who had previously plateaued for $\geq 6$ months on other hair loss treatments [14]. Starace et al. showed that the addition of $\mathrm{MN}$ improved hair counts in those already using hair loss treatments for $>1$ year (Table 2) [22].

\section{Adverse Events}

Across 536 subjects receiving $\mathrm{MN}$, no serious adverse events were reported. Of mild adverse 
events, transient pain, scalp irritation, and mild erythema were most commonly reported. Withdrawal rates across $\mathrm{MN}$ groups were low and comparable to non-MN groups.

\section{$\mathrm{AA}$ and $\mathrm{AT}$}

\section{Patients}

Of the five studies with AA and AT subjects, enrollment ages ranged from 16 to 45 years, with a subject-weighted average of 28.34 years. Three investigations enrolled subjects with hair loss gradients according to Severity Of Alopecia Tool (SALT) score, one study enrolled on the basis of severe AA, and one study enrolled on the basis of AT (Table 3).

\section{Interventions and Comparators}

In total, 114 subjects received $\mathrm{MN}$ therapy while 95 received other hair loss interventions. Of the five studies featuring AA and AT subjects, three compared treatments across patients $(n=181)$, while two compared treatments across lesions within the same patients $(n=28)$.

Across all study groups, MN was included as a standalone therapy in three groups $(n=68)$. As an adjunct therapy, $\mathrm{MN}$ was evaluated alongside a PRP topical in one group $(n=20)$, and with 5-aminolevulinic acid or methyl 5-aminolevulinic acid alongside photodynamic therapy in two groups $(n=25)$. As a comparator, $\mathrm{MN}$ was included as a control against cryotherapy in one group $(n=40)$, PRP injections in one group $(n=20)$, fractional $\mathrm{CO}_{2}$ laser alongside a PRP topical in one group $(n=20)$, triamcinolone acetonide injections in one group $(n=20), 5 \%$ minoxidil injections in one group $(n=20)$, and 5-aminolevulinic acid or methyl 5-aminolevulinic acid alongside photodynamic therapy in two groups $(n=23)$ (Table 3).

\section{MN Procedures}

MN devices tested included manual rollers $(n=3)$ and automated pens $(n=2)$. Needle lengths ranged from 1.00 to $5.00 \mathrm{~mm}$, with a mean needle length of $2.25 \mathrm{~mm}$.

The frequency of MN sessions ranged from once every 2 weeks to once monthly, with a mean session frequency of once every 3.46 weeks. The number of MN sessions ranged from three to six, with a mean of $4.40 \mathrm{MN}$ sessions per study. Treatment durations averaged 14.20 weeks.

While one study did not specify MN session endpoints, four studies standardized endpoints by a number of passes $(n=2)$, a number of passes and/or mild erythema $(n=1)$, or minutes of passes in affected areas $(n=1)$ (Table 3$)$.

\section{Outcomes}

In total, two of five studies assessed hair parameters through objective measurements (i.e., phototrichograms or $4 \mathrm{~mm}$ punch biopsies). Subjective measurements for the remaining three studies included Severity of Alopecia Tool (SALT) scores $(n=2)$, Lesional Area \& Density (LAD) scores $(n=1)$, and/or a fourpoint scale $(n=1)$.

Of the three studies testing standalone $\mathrm{MN}$ groups, Aboeldahab et al. and Abdallah et al. showed that MN alone increased hair density and improved SALT scores, respectively $[28,30]$. However, Giorgio et al. demonstrated no changes to hair parameters using a four-point scale to evaluate $\mathrm{MN}$ alone [31].

When comparing $\mathrm{MN}$ with cryotherapy, Aboeldahab et al. found significant increases to hair counts and hair densities across both interventions, with $\mathrm{MN}$ demonstrating greater changes to SALT scores versus cryotherapy [28]. Conversely, Abdallah et al. found that triamcinolone acetonide injections with and without $5 \%$ intradermal minoxidil led to greater improvements to SALT and LAD scores versus controls than $\mathrm{MN}$ alone [30].

As an adjunct therapy, Giorgio et al. showed that $\mathrm{MN}$ alongside 5-aminolevulinic acid and photodynamic therapy improved $94 \%$ of AA lesions versus $53 \%$ of lesions receiving only 5-aminolevulinic acid and photodynamic therapy [31]. However, Yoo et al. found that in AT subjects, methyl 5-aminolevulinic acid and photodynamic therapy with and without MN led to no hair parameter improvements according to $4 \mathrm{~mm}$ punch biopsies [32].

Finally, Ragab et al. demonstrated that, over a 3-month period, MN alongside topical PRP improves SALT scores similarly to fractional 


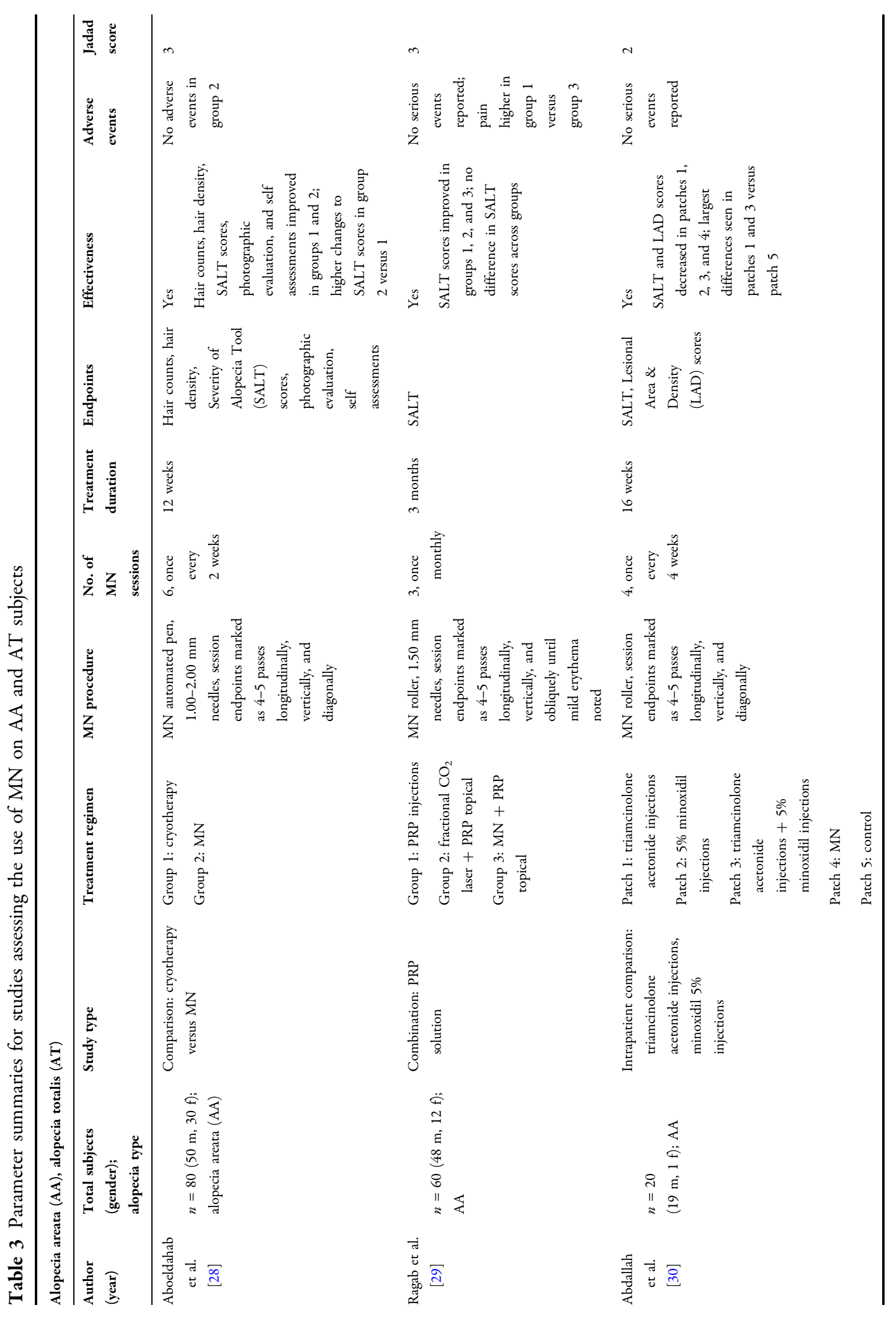




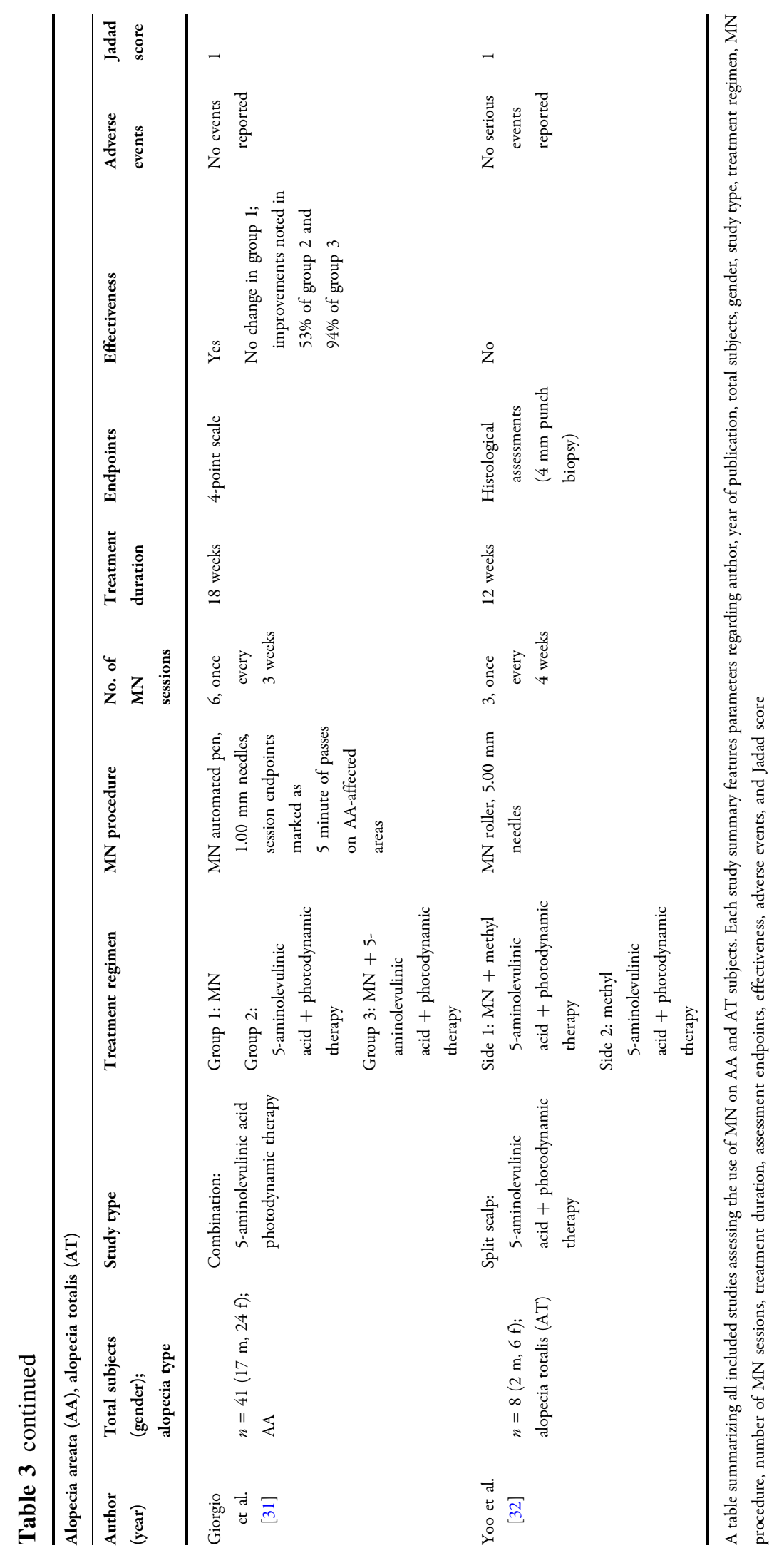


$\mathrm{CO}_{2}$ laser therapy alongside topical PRP (Table 3) [29].

\section{Adverse Events}

Among 114 subjects receiving $\mathrm{MN}$, no serious adverse events were reported. Of mild adverse events, transient pain and mild erythema were most common. Of the five studies, no withdrawals were reported in $\mathrm{MN}$ or non-MN groups.

\section{DISCUSSION}

Clinical studies demonstrate generally favorable results for $\mathrm{MN}$ as an adjunct therapy for AGA and AA. However, data are of relatively low quality and should be interpreted with caution. Due to significant heterogeneity across interventions, comparators, and $\mathrm{MN}$ procedures (i.e., devices, needle lengths, session frequencies, and session endpoints), we could not conduct a meta-analysis. Here we discuss the proposed mechanisms of $\mathrm{MN}$, limitations in the current body of research, and design considerations for future studies.

\section{Mechanisms}

\section{$A G A$}

In AGA-affected hair follicles, dihydrotestosterone dysregulates the $\mathrm{Wnt} / \beta$-catenin pathway, induces transforming growth factor $\beta 1$, and triggers apoptosis in dermal papillae and epithelial cells. This leads to a shortened anagen phase, reductions to dermal papillae cell cluster sizes with each re-entry into anagen and, consequently, microvascular degradation alongside progressive hair follicle miniaturization [33-36]. In mid-to-late stages of miniaturization, perifollicular fibrosis is often observed and may reduce the effectiveness of both systemic and topical AGA treatments [37].

As a monotherapy for AGA, data on $\mathrm{MN}$ are limited, and the mechanisms by which $\mathrm{MN}$ might improve AGA remain speculative. In a pooled linear regression across six subgroups, Gupta et al. found that MN significantly increased total hair counts, by more than $5 \%$ topical minoxidil [38]. However, two of the subgroups were a part of split-scalp studies assessing $\mathrm{MN}$ versus PRP injections or topical growth factor solutions [20, 27]. Therefore, the possibility of percutaneous treatment diffusion across scalp zones cannot be discounted.

Animal models suggest that MN may promote anagen-initiating $\mathrm{Wnt} / \beta$-catenin signaling and dermal papillae stem cell proliferation. In particular, percutaneous wounds from $\mathrm{MN}$ appear to activate hair follicle stem cells, platelet-derived growth factor, and vascular endothelial growth factor-potentiating the initiation of angiogenesis, neocollagenesis, and a new anagen cycle $[5-7,9,10]$. Clinical data show that $\mathrm{MN}$ reduces scarring and improves the density and thickness of epidermal and dermal skin layers $[39,40]$. In two randomized controlled clinical trials, Bao et al. found that MN alone increased terminal hair counts-with their latter study analyzing biopsies from a subset of AGA subjects showing that $\mathrm{MN}$ alone upregulated protein levels of both FZD3 and LEF-1 but not $\beta$-catenin $[19,25]$. However, RTPCR testing revealed no statistical increases to mRNA expression-with the authors postulating that the inconsistent results might be due to small sample sizes, infrequent needling sessions, shallow needling depths, and/or posttranscriptional modifications [25].

As an adjunct therapy, MN may improve AGA by enhancing transdermal delivery, and by improving sulfation and Wnt pathway expression when paired with topical minoxidil. Henry et al. demonstrated in vitro that $0.15 \mathrm{~mm}$ needles inserted into human skin for 10 seconds enhanced transdermal permeability of calcein by more than 1000-fold [41]. These effects may partly explain the equivalent and/or additive improvements to hair parameters from intradermal growth factors or PRP injections versus $\mathrm{MN}$ alongside their topical applications $[16,17]$. Additionally, MN may enhance topical minoxidil activation. Topical minoxidil is a pro-drug that requires sulfation by sulfotransferase enzymes in the outer root sheath of hair follicles [8]. Goren et al. demonstrated that reduced sulfotransferase activity in hair follicles predicted topical minoxidil nonresponders [42]. More recently, Sharma et al. found that, over 
21 days, once-weekly microneedling led to a median increase in sulfotransferase activity of $37.5 \%$ [8]. Finally, Bao et al. demonstrated that MN with 5\% minoxidil upregulated the expression of FZD3, LEF-1, and $\beta$-catenin in mRNA and protein more than $\mathrm{MN}$ or $5 \%$ minoxidil monotherapy-suggesting that the addition of MN might amplify the effects of minoxidil on the Wnt pathway [19].

\section{$A A$ and $A T$}

AA and AT are autoimmune forms of alopecia resulting from the collapse of immune privilege in affected hair follicles. In particular, peribulbar lymphocytic infiltrates appear to induce apoptosis in hair follicle keratinocytes-leading to inflammation, impaired hair shaft production, and sometimes hair shaft miniaturization within the same hair cycle. While the histologic features of AA and AT are well studied, their underlying pathogenesis remain poorly understood-with researchers speculating the involvement of immunological shifts related to genetic and environmental factors [4].

As a monotherapy for AA and AT, data on $\mathrm{MN}$ are unrobust. While Aboeldahab et al. and Abdallah et al. noted improvements to AA from $\mathrm{MN}$ alone, these results should be interpreted with caution due to relatively small sample sizes, as well as the $50 \%$ spontaneous recoveries observed in many AA studies with adequate controls [28, 30]. Moreover, Giorgio et al. found no effect from $\mathrm{MN}$ alone in AA subjects [31]. As an adjunct therapy, Giorgio et al. suggested that the release of growth factors from $\mathrm{MN}$ may induce immunosuppressive actions that amplify the effects of substances such as 5-aminolevulinic acid [31]. Ragab et al. found that MN alongside topical PRP improved hair parameters similarly to PRP injections, and suggested that MN may also enhance transdermal delivery for AA [29].

\section{Limitations}

Due to significant heterogeneity across $\mathrm{MN}$ studies regarding interventions, comparators, $\mathrm{MN}$ devices, needle lengths, session frequencies, and session endpoints, our systematic review does not include a meta-analysis and cannot establish best practices for MN procedures.

Faghihi et al. found hair parameters improved more when pairing 5\% minoxidil with fortnightly MN using an automated pen with needle lengths of $0.60 \mathrm{~mm}$ versus $1.20 \mathrm{~mm}$ [21]. Faghihi et al. postulated that puncture depths of $0.60 \mathrm{~mm}$ still generate enough of an inflammatory response for stem cell and growth factor recruitment, but without damaging the hair follicle bulge residing $1.00-1.80 \mathrm{~mm}$ from the skin surface [43]. Interestingly, Sasaki found that with MN automated pens, needling lengths matched penetration depths up to $1.50 \mathrm{~mm}$ [44]. Due to user pressure variability and needle entry angulation, Lima et al. estimated that a $3.00 \mathrm{~mm} \mathrm{MN}$ manual roller only penetrates to skin depths $50-70 \%$ of its needle length [45]. Taken together, equivalent $\mathrm{MN}$ penetration depths of $0.60-0.80 \mathrm{~mm}$ may be achievable with $\mathrm{MN}$ automated pens and $\mathrm{MN}$ manual rollers set to needle lengths of $0.60-0.80 \mathrm{~mm}$ and $1.25-1.50 \mathrm{~mm}$, respectively. Relatedly, Fernandes postulated that $\mathrm{MN}$ device preferences do not matter so long as the skin is penetrated to the same depths [46]. Regardless of standardizations for $\mathrm{MN}$ devices or needle lengths, additional methodological considerations-i.e., session durations, frequencies, and endpointsstill likely exert influence over the degree of inflammation induced, and thereby the magnitude of outcomes across a variety of hair parameters. As such, no procedural best practices can be ascertained with the current body of evidence.

While 8 of 22 clinical studies on MN included groups to evaluate $\mathrm{MN}$ alone $(n=174)$, only 1 study compared MN monotherapy against an untreated control patch for AA $(n=20)$. Moreover, 21 of 22 clinical studies on $\mathrm{MN}$ assessed hair parameter changes over periods of less than 52 weeks. Study durations of less than 52 weeks often do not allow investigators to separate the effects of any intervention against seasonal fluctuations to hair cyclingparticularly in the absence of untreated control groups $[47,48]$.

Finally, 4 out of 22 clinical studies utilized split-scalp study designs to evaluate $\mathrm{MN}$ against or as an adjuvant to topicals and/or injectables. 
Since MN is suspected to enhance transdermal drug delivery, split-scalp study designs leave open the possibility of percutaneous drug diffusion across scalp zones, thus limiting the interpretability of endpoint assessments.

\section{Recommendations}

Large-scale, randomized, placebo-controlled clinical trials assessing the use of $\mathrm{MN}$ for hair loss are needed. Future investigations should consider study durations of at least 12 months, include groups for $\mathrm{MN}$ as a monotherapy, and evaluate $\mathrm{MN}$ against a placebo (i.e., manual rollers with removed needles, automated pens with uninstalled needle cartridges, and/or an untreated control group). Split-scalp studies should be avoided, particularly when evaluating MN against or as an adjuvant to topicals and/or injectables. Finally, studies evaluating the use of $\mathrm{MN}$ across different procedural standards (i.e., shorter needle lengths and more frequent sessions versus longer needle lengths and less frequent sessions) will help toward establishing best practices.

\section{CONCLUSION}

Among 22 clinical studies featuring 1127 subjects, $\mathrm{MN}$ as an adjunct therapy improved hair parameters across genders as well as a range of hair loss types, hair loss severities, needling devices, needling depths of $0.50-2.50 \mathrm{~mm}$, and session frequencies from once weekly to once monthly-with no serious adverse events reported. However, results should be interpreted with caution due to significant heterogeneity across study interventions, comparators, and MN procedures (i.e., devices, needle lengths, session frequencies, and session endpoints). Large-scale randomized controlled trials are needed to discern the effects of $\mathrm{MN}$ as a standalone and adjunct therapy, determine best practices for $\mathrm{MN}$ procedures, and establish longterm safety data. Study designs should consider 12-month durations, include groups using MN as a monotherapy, and evaluate MN against a placebo and/or untreated group.

\section{ACKNOWLEDGEMENTS}

Funding. No funding or sponsorship was received for this study or publication of this article.

Authorship. All named authors meet the International Committee of Medical Journal Editors (ICMJE) criteria for authorship for this article, take responsibility for the integrity of the work as a whole, and have given their approval for this version to be published.

Author Contributions. RE contributed to the concept/design, systematic review process, and writing of the manuscript. RE, SR, and PD contributed to the systematic review process.

Disclosures. Robert S. English, Sophia Ruiz, and Pedro DoAmaral have nothing to disclose.

Compliance with Ethics Guidelines. This article is based on previously conducted studies and does not contain any new studies with human participants or animals performed by any of the authors.

Data Availability. Data availability is not applicable to this article as no datasets were generated or analyzed during the current study.

Open Access. This article is licensed under a Creative Commons Attribution-NonCommercial 4.0 International License, which permits any non-commercial use, sharing, adaptation, distribution and reproduction in any medium or format, as long as you give appropriate credit to the original author(s) and the source, provide a link to the Creative Commons licence, and indicate if changes were made. The images or other third party material in this article are included in the article's Creative Commons licence, unless indicated otherwise in a credit line to the material. If material is not included in the article's Creative Commons licence and your intended use is not permitted by statutory regulation or exceeds the permitted use, you will need to obtain permission directly from the copyright holder. To view a copy of this licence, 
visit http://creativecommons.org/licenses/bync/4.0/.

\section{REFERENCES}

1. Gan DC, Sinclair RD. Prevalence of male and female pattern hair loss in Maryborough. J Investig Dermatol Symp Proc. 2005;10(3):184-9.

2. Vidal CI. Overview of alopecia: a dermatopathologist's perspective. Mo Med. 2015;112(4):308-12.

3. Kaufman KD, Rotonda J, Shah AK, Meehan AG. Long-term treatment with finasteride $1 \mathrm{mg}$ decreases the likelihood of developing further visible hair loss in men with androgenetic alopecia (male pattern hair loss). Eur J Dermatol. 2008;18(4):400-6.

4. Trüeb RM, Dias MFRG. Alopecia areata: a comprehensive review of pathogenesis and management. Clin Rev Allergy Immunol. 2018;54(1):68-87.

5. Orentreich DS, Orentreich N. Subcutaneous incisionless (subcision) surgery for the correction of depressed scars and wrinkles. Dermatol Surg. 1995;21(6):543-9.

6. Iriarte C, Awosika O, Rengifo-Pardo M, Ehrlich A. Review of applications of microneedling in dermatology. Clin Cosmet Investig Dermatol. 2017;10: 289-98.

7. Ziaeifar E, Ziaeifar F, Mozafarpoor S, Goodarzi A. Applications of microneedling for various dermatologic indications with a special focus on pigmentary disorders: A comprehensive review study. Dermatol Ther. 2021:e15159.

8. Sharma A, Surve R, Dhurat R, Sinclair R, Tan T, Zou $\mathrm{Y}$, et al. Microneedling improves minoxidil response in androgenetic alopecia patients by upregulating follicular sulfotransferase enzymes. J Biol Regul Homeost Agents. 2020;34(2):659-61.

9. Kim YS, Jeong KH, Kim JE, Woo YJ, Kim BJ, Kang H. Repeated microneedle stimulation induces enhanced hair growth in a murine model. Ann Dermatol. 2016;28(5):586-92.

10. Ito M, Yang Z, Andl T, Cui C, Kim N, Millar SE, et al. Wnt-dependent de novo hair follicle regeneration in adult mouse skin after wounding. Nature. 2007;447(7142):316-20.

11. Dhurat R, Sukesh M, Avhad G, Dandale A, Pal A, Pund P. A randomized evaluator blinded study of effect of microneedling in androgenetic alopecia: a pilot study. Int J Trichol. 2013;5(1):6-11.
12. Ramadan WM, Hassan AM, Ismail MA, El Attar YA. Evaluation of adding platelet-rich plasma to combined medical therapy in androgenetic alopecia. J Cosmet Dermatol. 2021;20(5):1427-34.

13. Sohng C, Lee EH, Woo SK, Kim JY, Park KD, Lee SJ, et al. Usefulness of home-use microneedle devices in the treatment of pattern hair loss. J Cosmet Dermatol. 2021;20(2):591-6.

14. Burns LJ, Hagigeorges D, Flanagan KE, Pathoulas J, Senna MM. A pilot evaluation of scalp skin wounding to promote hair growth in female pattern hair loss. Int J Womens Dermatol. 2021;7(3): 344-5.

15. Gowda A, Sankey S, Kumar S. Comparative study of efficacy of minoxidil versus minoxidil with platelet rich plasma versus minoxidil with dermaroller in androgenetic alopecia. Int $\mathrm{J}$ Res Dermatol. 2021;7(2):279.

16. Shome D, Kapoor R, Vadera S, Doshi K, Patel G, Mohammad KT. Evaluation of efficacy of intradermal injection therapy vs derma roller application for administration of QR678 Neo. J Cosmet Dermatol. 2021;20(10):3299-307.

17. Ozcan KN, Sener S, Altunisik N, Turkmen D. PRP application by dermapen microneedling and intradermal point-by-point injection methods, and their comparison with clinical findings and trichoscan in patients. Dermatol Ther. 2021. https://doi.org/10. $1111 /$ dth.15182.

18. Yu CQ, Zhang H, Guo ME, Li XK, Chen HD, Li YH, et al. Combination therapy with topical minoxidil and nano-microneedle-assisted fibroblast growth factor for male androgenetic alopecia: a randomized controlled trial in Chinese patients. Chin Med J (Engl). 2020;134(7):851-3.

19. Bao L, Zong H, Fang S, Zheng L, Li Y. Randomized trial of electrodynamic microneedling combined with $5 \%$ minoxidil topical solution for treating androgenetic alopecia in Chinese males and molecular mechanistic study of the involvement of the Wnt/ $\beta$-catenin signaling pathway. J Dermatol Treat. 2020. https://doi.org/10.1080/09546634. 2020.1770162 .

20. Aggarwal K, Gupta S, Jangra RS, Mahendra A, Yadav A, Sharma A. Dermoscopic assessment of microneedling alone versus microneedling with plateletrich plasma in cases of male pattern alopecia: a split-head comparative study. Int $\mathrm{J}$ Trichol. 2020;12(4):156-63.

21. Faghihi G, Nabavinejad S, Mokhtari F, Fatemi Naeini F, Iraji F. Microneedling in androgenetic alopecia; comparing two different depths of 
microneedles. J Cosmet Dermatol. 2021;20(4): 1241-7.

22. Starace M, Alessandrini A, Brandi N, Piraccini BM. Preliminary results of the use of scalp microneedling in different types of alopecia. J Cosmet Dermatol. 2020;19(3):646-50.

23. Kumar MK, Inamadar AC, Palit A. A randomized controlled, single-observer blinded study to determine the efficacy of topical minoxidil plus microneedling versus topical minoxidil alone in the treatment of androgenetic alopecia. J Cutan Aesthet Surg. 2018;11(4):211-6.

24. Yu AJ, Luo YJ, Xu XG, Bao LL, Tian T, Li ZX, et al. A pilot split-scalp study of combined fractional radiofrequency microneedling and 5\% topical minoxidil in treating male pattern hair loss. Clin Exp Dermatol. 2018;43(7):775-81.

25. Bao L, Gong L, Guo M, Liu T, Shi A, Zong H, et al. Randomized trial of electrodynamic microneedle combined with $5 \%$ minoxidil topical solution for the treatment of Chinese male androgenetic alopecia. J Cosmet Laser Ther. 2020;22(1):1-7.

26. Shah KB, Shah AN, Solanki RB, Raval RC. A comparative study of microneedling with platelet-rich plasma plus topical minoxidil (5\%) and topical minoxidil (5\%) alone in androgenetic alopecia. Int J Trichol. 2017;9(1):14-8.

27. Lee YB, Eun YS, Lee JH, Cheon MS, Park YG, Cho $\mathrm{BK}$, et al. Effects of topical application of growth factors followed by microneedle therapy in women with female pattern hair loss: a pilot study. J Dermatol. 2013;40(1):81-3.

28. Aboeldahab S, Nada EEA, Assaf HA, Gouda ZA, Abu El-Hamd M. Superficial cryotherapy using dimethyl ether and propane mixture versus microneedling in the treatment of alopecia areata: a prospective single-blinded randomized clinical trial. Dermatol Ther. 2021;34(5): e15044.

29. Ragab SEM, Nassar SO, Morad HA, Hegab DS. Platelet-rich plasma in alopecia areata: intradermal injection versus topical application with transepidermal delivery via either fractional carbon dioxide laser or microneedling. Acta Dermatovenerol Alp Pannonica Adriat. 2020;29(4):169-73.

30. Abdallah MAE, Shareef R, Soltan MY. Efficacy of intradermal minoxidil 5\% injections for treatment of patchy non-severe alopecia areata. J Dermatol Treat. 2020. https://doi.org/10.1080/09546634. 2020.1793893.

31. Giorgio CM, Babino G, Caccavale S, Russo T, De Rosa AB, Alfano R, et al. Combination of photodynamic therapy with 5-aminolaevulinic acid and microneedling in the treatment of alopecia areata resistant to conventional therapies: our experience with 41 patients. Clin Exp Dermatol. 2020;45(3): 323-6.

32. Yoo KH, Lee JW, Li K, Kim BJ, Kim MN. Photodynamic therapy with methyl 5-aminolevulinate acid might be ineffective in recalcitrant alopecia totalis regardless of using a microneedle roller to increase skin penetration. Dermatol Surg. 2010;36(5): 618-22.

33. Inui S, Fukuzato Y, Nakajima T, Yoshikawa K, Itami S. Androgen-inducible TGF-beta1 from balding dermal papilla cells inhibits epithelial cell growth: a clue to understand paradoxical effects of androgen on human hair growth. FASEB J. 2002;16(14): 1967-9.

34. Inui S, Fukuzato Y, Nakajima T, Yoshikawa K, Itami S. Identification of androgen-inducible TGF- $\beta 1$ derived from dermal papilla cells as a key mediator in androgenetic alopecia. J Investig Dermatol Symp Proc. 2003;8(1):69-71.

35. Winiarska A, Mandt N, Kamp H, Hossini A, Seltmann $\mathrm{H}$, Zouboulis CC, et al. Effect of $5 \alpha$-dihydrotestosterone and testosterone on apoptosis in human dermal papilla cells. Skin Pharmacol Physiol. 2006;19(6):311-21.

36. Lolli F, Pallotti F, Rossi A, Fortuna MC, Caro G, Lenzi A, et al. Androgenetic alopecia: a review. Endocrine. 2017;57(1):9-17.

37. English R, Ruiz S. Conflicting reports regarding the histopathological features of androgenic alopecia: Are biopsy location, hair diameter diversity, and relative hair follicle miniaturization partly to blame? Clin Cosmet Investig Dermatol. 2021;14: 357-65.

38. Gupta AK, Quinlan EM, Venkataraman M, Bamimore MA. Microneedling for hair loss. J Cosmet Dermatol. 2021. https://doi.org/10.1111/jocd. 14525.

39. Alster TS, Li MKY. Microneedling of scars: a large prospective study with long-term follow-up. Plast Reconstr Surg. 2020;145(2):358-64.

40. El-Domyati M, Barakat M, Awad S, Medhat W, ElFakahany H, Farag H. Microneedling therapy for atrophic acne scars: an objective evaluation. J Clin Aesthet Dermatol. 2015;8(7):36-42.

41. Henry S, McAllister DV, Allen MG, Prausnitz MR. Microfabricated microneedles: a novel approach to transdermal drug delivery. J Pharm Sci. 1998;87(8): $922-5$. 
42. Goren A, Castano JA, McCoy J, Bermudez F, Lotti T. Novel enzymatic assay predicts minoxidil response in the treatment of androgenetic alopecia. Dermatol Ther. 2014;27(3):171-3.

43. Jimenez F, Izeta A, Poblet E. Morphometric analysis of the human scalp hair follicle: practical implications for the hair transplant surgeon and hair regeneration studies. Dermatol Surg. 2011;37(1): $58-64$.

44. Sasaki GH. Micro-needling depth penetration, presence of pigment particles, and fluoresceinstained platelets: clinical usage for aesthetic concerns. Aesthet Surg J. 2017;37(1):71-83.
45. Lima EVdA, Lima MdA, Takano D. Microneedling: experimental study and classification of the resulting injury. Surg Cosmet Dermatol; 2013;110-11-4.

46. Fernandes D. Commentary on: micro-needling depth penetration, presence of pigment particles, and fluorescein-stained platelets: clinical usage for aesthetic concerns. Aesthet Surg J. 2017;37(1):86-8.

47. Randall VA, Ebling FJ. Seasonal changes in human hair growth. Br J Dermatol. 1991;124(2):146-51.

48. Trüeb RM. Telogen effluvium: Is there a need for a new classification? Skin Appendage Disord. 2016;2(1-2):39-44. 